\title{
Improved Language Modeling by Decoding the Past
}

\author{
Siddhartha Brahma \\ IBM Research, Almaden, USA \\ brahma@us.ibm.com
}

\begin{abstract}
Highly regularized LSTMs achieve impressive results on several benchmark datasets in language modeling. We propose a new regularization method based on decoding the last token in the context using the predicted distribution of the next token. This biases the model towards retaining more contextual information, in turn improving its ability to predict the next token. With negligible overhead in the number of parameters and training time, our Past Decode Regularization (PDR) method improves perplexity on the Penn Treebank dataset by up to 1.8 points and by up to 2.3 points on the WikiText-2 dataset, over strong regularized baselines using a single softmax. With a mixture-of-softmax model, we show gains of up to 1.0 perplexity points on these datasets. In addition, our method achieves 1.169 bits-per-character on the Penn Treebank Character dataset for character level language modeling. Each of these results constitute improvements over models without PDR in their respective settings.
\end{abstract}

\section{Introduction}

Language modeling is a fundamental task in natural language processing. Given a sequence of tokens, its joint probability distribution can be modeled using the auto-regressive conditional factorization. This leads to a convenient formulation where a language model has to predict the next token given a sequence of tokens as context. Recurrent neural networks are an effective way to compute distributed representations of the context by sequentially operating on the embeddings of the tokens. These representations can then be used to predict the next token as a probability distribution over a fixed vocabulary using a linear decoder followed by Softmax.

Starting from the work of (Mikolov et al., 2010), there has been a long list of works that seek to improve language modeling performance using more sophisticated recurrent neural networks (RNNs) (Zaremba et al., 2014; Zilly et al., 2017; Zoph and Le, 2016; Mujika et al., 2017). However, in more recent work vanilla LSTMs (Hochreiter and Schmidhuber, 1997) with relatively large number of parameters have been shown to achieve state-of-the-art performance on several standard benchmark datasets both in wordlevel and character-level perplexity (Merity et al., 2018b,a; Melis et al., 2018; Yang et al., 2017). A key component in these models is the use of several forms of regularization e.g. variational dropout on the token embeddings ( $\mathrm{Gal}$ and Ghahramani, 2016), dropout on the hidden-tohidden weights in the LSTM (Wan et al., 2013), norm regularization on the outputs of the LSTM and classical dropout (Srivastava et al., 2014). By carefully tuning the hyperparameters associated with these regularizers combined with optimization algorithms like NT-ASGD (a variant of Averaged SGD), it is possible to achieve very good performance. Each of these regularizations address different parts of the LSTM model and are general techniques that could be applied to any other sequence modeling problem.

In this paper, we propose a regularization technique that exploits a symmetry in language models. A unique aspect of language modeling using LSTMs (or any RNN) is that at each time step $t$, the model takes as input a particular token $x_{t}$ from a vocabulary $W$ and using the hidden state of the LSTM (which encodes the context till $x_{t-1}$ ) predicts a probability distribution $\mathbf{w}_{t+1}$ on the next token $x_{t+1}$ over the same vocabulary as output. Since $x_{t}$ can be mapped to a trivial probability distribution over $W$, this operation can be interpreted as transforming distributions over $W$ (Inan et al., 2016). Clearly, the output distribution is dependent on and is a function of $x_{t}$ and the context 
further in the past and encodes information about it. We ask the following question - How much information is it possible to decode about the input distribution (and hence $x_{t}$ ) from the output distribution $\mathbf{w}_{t+1}$ ? In general, it is impossible to decode $x_{t}$ unambiguously. Even if the language model is perfect and correctly predicts $x_{t+1}$ with probability 1 , there could be many tokens preceding it. However, in this case the number of possibilities for $x_{t}$ will be limited, as dictated by the bigram statistics of the corpus and the language in general. We argue that biasing the language model such that it is possible to decode more information about the past tokens from the predicted next token distribution is beneficial. We incorporate this intuition into a regularization term in the loss function of the language model.

The symmetry in the inputs and outputs of the language model at each step lends itself to a simple decoding operation. It can be cast as a (pseudo) language modeling problem in "reverse", where the future prediction $\mathbf{w}_{t+1}$ acts as the input and the last token $x_{t}$ acts as the target of prediction. The token embedding matrix and weights of the linear decoder of the main language model can be reused in the past decoding operation. We only need a few extra parameters to model the nonlinear transformation performed by the LSTM, which we do by using a simple stateless layer. We compute the cross-entropy loss between the decoded distribution for the past token and $x_{t}$ and add it to the main loss function after suitable weighting. The extra parameters used in the past decoding are discarded during inference time. We call our method Past Decode Regularization or PDR for short.

We conduct extensive experiments on four benchmark datasets for word level and character level language modeling by combining PDR with existing LSTM based language models and achieve improved performance on three of them.

\section{Past Decode Regularization (PDR)}

Let $\mathbf{X}=\left(x_{1}, x_{2}, \cdots, x_{t}, \cdots, x_{T}\right)$ be a sequence of tokens. In this paper, we will experiment with both word level and character level language modeling. Therefore, tokens can be either words or characters. The joint probability $P(\mathbf{X})$ factorizes into

$$
P(\mathbf{X})=\prod_{t=1}^{T} P\left(x_{t} \mid x_{1}, x_{2}, \cdots, x_{t-1}\right)
$$

Let $c_{t}=\left(x_{1}, x_{2}, \cdots, x_{t}\right)$ denote the context available to the language model for $x_{t+1}$. Let $W$ denote the vocabulary of tokens, each of which is embedded into a vector of dimension $d$. Let $\mathbf{E}$ denote the token embedding matrix of dimension $|W| \times d$ and $\mathbf{e}_{w}$ denote the embedding of $w \in W$. An LSTM computes a distributed representation of $c_{t}$ in the form of its hidden state $\mathbf{h}_{t}$, which we assume has dimension $d$ as well. The probability that the next token is $w$ can then be calculated using a linear decoder followed by a Softmax layer as

$$
\begin{aligned}
P_{\theta}\left(w \mid c_{t}\right) & =\left.\operatorname{Smax}\left(\mathbf{h}_{t} \mathbf{E}^{\mathrm{T}}+\mathbf{b}\right)\right|_{w} \\
& =\frac{\exp \left(\mathbf{h}_{t} \mathbf{e}_{w}^{\mathrm{T}}+b_{w}\right)}{\sum_{w^{\prime} \in W} \exp \left(\mathbf{h}_{t} \mathbf{e}_{w^{\prime}}^{\mathrm{T}}+b_{w^{\prime}}\right)}
\end{aligned}
$$

where $b_{w^{\prime}}$ is the entry corresponding to $w^{\prime}$ in a bias vector $\mathbf{b}$ of dimension $|W|$ and $\left.\right|_{w}$ represents projection onto $w$. Here we assume that the weights of the decoder are tied with the token embedding matrix E (Inan et al., 2016; Press and Wolf, 2017). To optimize the parameters of the language model $\theta$, the loss function to be minimized during training is set as the cross-entropy between the predicted distribution $P_{\theta}\left(w \mid c_{t}\right)$ and the actual token $x_{t+1}$.

$$
\mathcal{L}_{C E}=\sum_{t}-\log \left(P_{\theta}\left(x_{t+1} \mid c_{t}\right)\right)
$$

Note that Eq.(2), when applied to all $w \in W$ produces a $1 \times|W|$ vector $\mathbf{w}_{t+1}$, encapsulating the prediction the language model has about the next token $x_{t+1}$. Since this is dependent on and conditioned on $c_{t}, \mathbf{w}_{t+1}$ clearly encodes information about it; in particular about the last token $x_{t}$ in $c_{t}$. In turn, it should be possible to infer or decode some limited information about $x_{t}$ from $\mathbf{w}_{t+1}$. We argue that by biasing the model to be more accurate in recalling information about past tokens, we can help it in predicting the next token better.

To this end, we define the following decoding operation to compute a probability distribution over $w_{c} \in W$ as the last token in the context.

$$
P_{\theta_{r}}\left(w_{c} \mid \mathbf{w}_{t+1}\right)=\left.\operatorname{Smax}\left(f_{\theta_{r}}\left(\mathbf{w}_{t+1} \mathbf{E}\right) \mathbf{E}^{\mathrm{T}}+\mathbf{b}_{\theta_{r}}^{\prime}\right)\right|_{w_{c}}
$$

Here $f_{\theta_{r}}$ is a non-linear function that maps vectors in $\mathbb{R}^{d}$ to vectors in $\mathbb{R}^{d}$ and $\mathbf{b}_{\theta_{r}}^{\prime}$ is a bias vector of dimension $|W|$, together comprising the parameters $\theta_{r}$. In effect, we are decoding the past - the last token in the context $x_{t}$. This produces a vector $\mathbf{w}_{t}^{r}$ of dimension $1 \times|W|$. The cross-entropy loss 


\begin{tabular}{|c|c|c|c|c|c|c|c|c|c|c|c|c|}
\hline & \multicolumn{3}{|c|}{ PTB } & \multicolumn{3}{|c|}{ WT2 } & \multicolumn{3}{|c|}{ РTBC } & \multicolumn{3}{|c|}{ enwik8 } \\
\hline & Train & Valid & Test & Train & Valid & Test & Train & Valid & Test & Train & Valid & Test \\
\hline $\begin{array}{l}\text { Tokens } \\
\text { Vocab }\end{array}$ & $888 \mathrm{~K}$ & $\begin{array}{c}70.4 \mathrm{~K} \\
10 \mathrm{~K}\end{array}$ & $78.7 \mathrm{~K}$ & $2.05 \mathrm{M}$ & $\begin{array}{r}213 \mathrm{~K} \\
33.3 \mathrm{~K}\end{array}$ & $241 \mathrm{~K}$ & $5.01 \mathrm{M}$ & $\begin{array}{c}393 \mathrm{k} \\
51\end{array}$ & $442 \mathrm{k}$ & $90 \mathrm{M}$ & $\begin{array}{l}5 \mathrm{M} \\
205\end{array}$ & $5 \mathrm{M}$ \\
\hline
\end{tabular}

Table 1: Statistics of the language modeling benchmark datasets.

with respect to the actual last token $x_{t}$ can then be computed as

$$
\mathcal{L}_{P D R}=\sum_{t}-\log \left(P_{\theta_{r}}\left(x_{t} \mid \mathbf{w}_{t+1}\right)\right)
$$

Here PDR stands for Past Decode Regularization. $\mathcal{L}_{P D R}$ captures the extent to which the decoded distribution of tokens differs from the actual tokens $x_{t}$ in the context. Note the symmetry between Eqs.(2) and (5). The "input" in the latter case is $\mathbf{w}_{t+1}$ and the "context" is provided by a nonlinear transformation of $\mathbf{w}_{t+1} \mathbf{E}$. Different from the former, the context in Eq.(5) does not preserve any state information across time steps as we want to decode only using $\mathbf{w}_{t+1}$. The term $\mathbf{w}_{t+1} \mathbf{E}$ can be interpreted as a "soft" token embedding lookup, where the token vector $\mathbf{w}_{t+1}$ is a probability distribution instead of a unit vector.

We add $\lambda_{P D R} \mathcal{L}_{P D R}$ to the loss function in Eq.(3) as a regularization term, where $\lambda_{P D R}$ is a positive weighting coefficient, to construct the following new loss function for the language model.

$$
\mathcal{L}=\mathcal{L}_{C E}+\lambda_{P D R} \mathcal{L}_{P D R}
$$

Thus equivalently PDR can also be viewed as a method of defining an augmented loss function for language modeling. The choice of $\lambda_{P D R}$ dictates the degree to which we want the language model to incorporate our inductive bias i.e. decodability of the last token in the context. If it is too large, the model will fail to predict the next token, which is its primary task. If it is zero or too small, the model will retain less information about the last token which hampers its predictive performance. In practice, we choose $\lambda_{P D R}$ by a search based on validation set performance.

Note that the trainable parameters $\theta_{r}$ associated with PDR are used only during training to bias the language model and are not used at inference time. This also means that it is important to control the complexity of the nonlinear function $f_{\theta_{r}}$ so as not to overly bias the training. As a simple choice, we use a single fully connected layer of size $d$ followed by a Tanh nonlinearity as $f_{\theta_{r}}$. This introduces few extra parameters and a small increase in training time as compared to a model not using PDR.

\section{Experiments}

We present extensive experimental results to show the efficacy of using PDR for language modeling on four standard benchmark datasets - two each for word level and character level language modeling. For the former, we evaluate our method on the Penn Treebank (PTB) (Mikolov et al., 2010) and the WikiText-2 (WT2) (Merity et al., 2016) datasets. For the latter, we use the Penn Treebank Character (PTBC) (Mikolov et al., 2010) and the Hutter Prize Wikipedia Prize (Hutter, 2018) (also known as Enwik8) datasets. Key statistics for these datasets is presented in Table 1 .

As mentioned in the introduction, some of the best existing results on these datasets are obtained by using extensive regularization techniques on relatively large LSTMs (Merity et al., 2018b,a; Yang et al., 2017). We apply our regularization technique to these models, the so called AWDLSTM. We consider two versions of the model one with a single softmax (AWD-LSTM) and one with a mixture-of-softmaxes (AWD-LSTM-MoS). The PDR regularization term is computed according to Eq.(4) and Eq.(5). We call our model AWDLSTM+PDR when using a single softmax and AWD-LSTM-MoS+PDR when using a mixtureof-softmaxes. We largely follow the experimental procedure of the original models and incorporate their dropouts and regularizations in our experiments.

For completeness, we briefly mention the set of dropouts and regularizations reused from AWDLSTM in our experiments. They are the following.

1. Embedding dropout - Variational or locked dropout applied to the token embedding matrix. 
2. Word dropout - Dropout applied to entire tokens.

3. LSTM layer dropout - Dropout between layers of the LSTM.

4. LSTM weight dropout - Dropout applied to the hidden-to-hidden connections in the LSTM.

5. LSTM output dropout - Dropout applied to the final output of the LSTM.

6. Alpha/beta regularization - Activation and temporal activation regularization applied to the LSTM states.

7. Weight decay - L2 regularization on the parameters of the model.

Note that these regularizations are applied to the input, hidden state and output of the LSTM and do not exploit the special structure of language modeling, which PDR does. The relative contribution of these existing regularizations and PDR will be analyzed in Section 6.

There are 7 hyperparameters associated with the regularizations used in AWD-LSTM (and one extra with MoS). PDR also has an associated weighting coefficient $\lambda_{P D R}$. For our experiments, we set $\lambda_{P D R}=0.001$ which was determined by a coarse search on the PTB and WT2 validation sets. For the remaining ones, we perform light hyperparameter search in the vicinity of those reported for AWD-LSTM in (Merity et al., 2018b,a) and for AWD-LSTM-MoS in (Yang et al., 2017).

\subsection{Model and training for PTB and WikiText-2}

For the single softmax model (AWDLSTM+PDR), for both PTB and WT2, we use a 3-layered LSTM with 1150, 1150 and 400 hidden dimensions. The word embedding dimension is set to $d=400$. For the mixture-of-softmax model, we use a 3-layer LSTM with dimensions 960, 960 and 620, an embedding dimension of 280 and 15 experts for PTB. For WT2, we use a 3-layer LSTM with dimensions 1150, 1150 and 650 , an embedding dimension of $d=300$ and 15 experts. Weight tying is used in all the models. For training the models, we follow the same procedure as AWD-LSTM i.e. a combination of SGD and NT-ASGD, followed by finetuning. We adopt the learning rate schedules and batch sizes of (Merity et al., 2018b) and (Yang et al., 2017) in our experiments.

\subsection{Model and training for PTBC and Enwik8}

For PTBC, we use a 3-layer LSTM with 1000, 1000 and 200 hidden dimensions and a character embedding dimension of $d=200$. For Enwik8, we use a LSTM with 1850,1850 and 400 hidden dimensions and the characters are embedded in $d=400$ dimensions. For training, we largely follow the procedure laid out in (Merity et al., 2018a). For each of the datasets, AWDLSTM+PDR has less than $1 \%$ more parameters than the corresponding AWD-LSTM model (during training only). The maximum observed time overhead due to the additional computation is less than $3 \%$.

\section{Results on Word Level Language Modeling}

The results for PTB are shown in Table 2. With a single softmax, our method (AWD-LSTM+PDR) achieves a perplexity of 55.6 on the PTB test set, which improves on the model without PDR by an absolute 1.7 points. The advantages of better information retention due to PDR are maintained when combined with a continuous cache pointer (Grave et al., 2016), where our method yields an absolute improvement of 1.2 over AWD-LSTM. Notably, when coupled with dynamic evaluation (Krause et al., 2018), the perplexity is decreased further to 49.3. Note that, for both cache pointer and dynamic evaluation, we coarsely tune the associated hyperparameters on the validation set.

Using a mixture-of-softmaxes, our method (AWD-LSTM-MoS+PDR) achieves a test perplexity of 53.8, an improvement of 0.6 points over the model without PDR. The use of dynamic evaluation pushes the perplexity further down to 47.3. Note that our models do not use the recently proposed frequency agnostic word embeddings FRAGE (Gong et al., 2018) and it is possible that adding PDR can lead to similar gains when applied to models using such embeddings. PTB is a restrictive dataset with a vocabulary of $10 \mathrm{~K}$ words. Achieving good perplexity requires considerable regularization. The fact that PDR can improve upon existing heavily regularized models is empirical evidence of its distinctive nature and its effectiveness in improving language models. 


\begin{tabular}{lccc}
\hline Model & \#Params & Valid & Test \\
\hline State-of-the-art Methods (Single Softmax) & & & \\
\hline (Merity et al., 2018b) - AWD-LSTM & $24.2 \mathrm{M}$ & 60.0 & 57.3 \\
(Merity et al., 2018b) - AWD-LSTM + continuous cache pointer & $24.2 \mathrm{M}$ & 53.9 & 52.8 \\
(Krause et al., 2018) - AWD-LSTM + dynamic evaluation & $24.2 \mathrm{M}$ & 51.6 & 51.1 \\
(Gong et al., 2018) - AWD-LSTM + cont. cache pointer w/ FRAGE & $24.2 \mathrm{M}$ & 52.3 & 51.8 \\
\hline Our Method (Single Softmax) & & & \\
\hline AWD-LSTM+PDR & $24.2 \mathrm{M}$ & 57.9 & $55.6(-1.7)$ \\
AWD-LSTM+PDR + continuous cache pointer & $24.2 \mathrm{M}$ & 52.4 & $51.6(-1.2)$ \\
AWD-LSTM+PDR + dynamic evaluation & $24.2 \mathrm{M}$ & 50.1 & $49.3(-\mathbf{1 . 8})$ \\
\hline Sate-of-the-art Methods (Mixture-of-Softmax) & & & \\
\hline (Yang et al., 2017) - AWD-LSTM-MoS & $22 \mathrm{M}$ & 56.5 & 54.4 \\
(Yang et al., 2017) - AWD-LSTM-MoS + dynamic evaluation & $22 \mathrm{M}$ & 48.3 & 47.7 \\
(Gong et al., 2018) - AWD-LSTM-MoS + dyn. evaluation w/ FRAGE & $22 \mathrm{M}$ & 47.4 & 46.5 \\
\hline Our Method (Mixture-of-Softmax) & & & \\
\hline AWD-LSTM-MoS+PDR & $22 \mathrm{M}$ & 56.2 & $53.8(-\mathbf{0 . 6})$ \\
AWD-LSTM-MoS+PDR + dynamic evaluation & $22 \mathrm{M}$ & 48.0 & $47.3(-\mathbf{0 . 4})$ \\
\hline
\end{tabular}

Table 2: Perplexities on Penn Treebank (PTB) for single and mixture-of-softmaxes models. Values in parentheses show gain over respective models without using PDR. The number of parameters during training is $24.4 \mathrm{M}$. Our models do not use frequency agnostic word embeddings.

Table 3 shows the perplexities achieved by our model on WT2. This dataset is considerably more complex than PTB with a vocabulary of more than $33 \mathrm{~K}$ words. AWD-LSTM+PDR improves over the single softmax model without PDR by a significant 2.3 points, achieving a perplexity of 63.5. Similar gains are observed with the use of cache pointer ( 2.4 points) and with the use of dynamic evaluation (1.7 points). Using a mixture-ofsoftmaxes, AWD-LSTM-MoS+PDR achieves perplexities of 60.5 and 40.3 (with dynamic evaluation) on the WT2 test set, improving upon the models without PDR by 1.0 and 0.4 points respectively. Here again, the use of PDR in models with FRAGE could lead to further drops in perplexity.

\subsection{Performance on Larger Datasets}

We consider the Gigaword dataset (Chelba et al., 2014 ) with a truncated vocabulary of about $100 \mathrm{~K}$ tokens with the highest frequency and apply PDR to a baseline 2-layer LSTM language model with embedding and hidden dimensions set to 1024 . We use all the shards from the training set for training and a few shards from the heldout set for validation (heldout- 0,10 ) and test (heldout20,30,40). We tuned the PDR coefficient coarsely in the vicinity of 0.001 . While the baseline model achieved a validation (test) perplexity of 44.3 (43.1), on applying PDR, the model achieved a perplexity of 44.0 (42.5). Thus, PDR is relatively less effective on larger datasets, a fact also observed for other regularization techniques on such datasets (Yang et al., 2017).

\section{Results on Character Level Language Modeling}

The results on PTBC are shown in Table 4. Our method achieves a bits-per-character (BPC) performance of 1.169 on the PTBC test set, improving on the model without PDR by 0.006 or $0.5 \%$. It is notable that even with this highly processed dataset and a small vocabulary of only 51 tokens, our method improves on already highly regularized models. Finally, we present results on Enwik8 in Table 5. AWD-LSTM+PDR achieves 1.245 BPC. This is 0.012 or about $1 \%$ less than the 1.257 BPC achieved by AWD-LSTM in our experiments (with hyperparameters from (Merity et al., 2018a).

\section{Analysis of PDR}

In this section, we analyze PDR by probing its performance in several ways and comparing it with models that do not use PDR.

\subsection{A Valid Regularization}

To verify that indeed PDR can act as a form of regularization, we perform the following exper- 


\begin{tabular}{llll}
\hline Model & \#Params & Valid & Test \\
\hline Sate-of-the-art Methods (Single Softmax) & & & \\
\hline (Merity et al., 2018b) - AWD-LSTM & $33.6 \mathrm{M}$ & 68.6 & 65.8 \\
(Merity et al., 2018b) - AWD-LSTM + continuous cache pointer & $33.6 \mathrm{M}$ & 53.8 & 52.0 \\
(Krause et al., 2018) - AWD-LSTM + dynamic evaluation & $33.6 \mathrm{M}$ & 46.4 & 44.3 \\
(Gong et al., 2018) - AWD-LSTM + cont. cache ptr w/ FRAGE & $33.6 \mathrm{M}$ & 51.0 & 49.3 \\
\hline Our Method (Single Softmax) & & & \\
\hline AWD-LSTM+PDR & $33.6 \mathrm{M}$ & 66.5 & $63.5(-2.3)$ \\
AWD-LSTM+PDR + continuous cache pointer & $33.6 \mathrm{M}$ & 51.5 & $49.6(-2.4)$ \\
AWD-LSTM+PDR + dynamic evaluation & $33.6 \mathrm{M}$ & 44.6 & $42.6(-1.7)$ \\
\hline Sate-of-the-art Methods (Mixture-of-Softmax) & & & \\
\hline (Yang et al., 2017) - AWD-LSTM-MoS & $35 \mathrm{M}$ & 63.9 & 61.5 \\
(Yang et al., 2017) - AWD-LSTM-MoS + dynamic evaluation & $35 \mathrm{M}$ & 42.4 & 40.7 \\
(Gong et al., 2018) - AWD-LSTM-MoS + dyn. eval w/ FRAGE & $35 \mathrm{M}$ & 40.9 & 39.1 \\
\hline Our Method (Mixture-of-Softmax) & & & \\
\hline AWD-LSTM-MoS+PDR & $35 \mathrm{M}$ & 63.0 & $60.5(-1.0)$ \\
AWD-LSTM-MoS+PDR + dynamic evaluation & $35 \mathrm{M}$ & 42.0 & $40.3(-\mathbf{0 . 4})$ \\
\hline
\end{tabular}

Table 3: Perplexities on WikiText-2 (WT2) for single and mixture-of-softmaxes models. Values in parentheses show gain over respective models without using PDR. The number of parameters during training is $33.8 \mathrm{M}$. We do not use frequency agnostic word embeddings.

\begin{tabular}{lll}
\hline Model & \#Prms & Test \\
\hline (Krueger et al., 2016) - Zoneout LSTM & - & 1.27 \\
(Chung et al., 2016) - HM-LSTM & - & 1.24 \\
(Ha et al., 2016) - HyperLSTM & $14.4 \mathrm{M}$ & 1.219 \\
(Zoph and Le, 2016) - NAS Cell & $16.3 \mathrm{M}$ & 1.214 \\
(Mujika et al., 2017) - FS-LSTM-4 & $6.5 \mathrm{M}$ & 1.193 \\
(Merity et al., 2018a) - AWD-LSTM & $13.8 \mathrm{M}$ & 1.175 \\
\hline Our Method & \multicolumn{2}{r}{} \\
\hline AWD-LSTM+PDR & $13.8 \mathrm{M}$ & 1.169 \\
& \multicolumn{2}{r}{$\mathbf{( - 0 . 0 0 6 )}$} \\
\hline
\end{tabular}

Table 4: Bits-per-character on the PTBC test set.

iment. We take the models for PTB and WT2 and turn off all dropouts and regularization and compare its performance with only PDR turned on. The results, as shown in Table 6, validate the premise of PDR. The model with only PDR turned on achieves 2.4 and 5.1 better validation perplexity on PTB and WT2 as compared to the model without any regularization. Thus, biasing the LSTM by decoding the distribution of past tokens from the predicted next-token distribution can indeed act as a regularizer leading to better generalization performance.

Next, in Fig. 1(a) we plot histograms of the negative log-likelihoods of the correct context tokens $x_{t}$ in the past decoded vector $\mathbf{w}_{t}^{r}$ computed using

\begin{tabular}{lll}
\hline Model & \#Prms & Test \\
\hline (Ha et al., 2016) - HyperLSTM & $27 \mathrm{M}$ & 1.340 \\
(Chung et al., 2016) - HM-LSTM & $35 \mathrm{M}$ & 1.32 \\
(Rocki et al., 2016) - SD Zoneout & $64 \mathrm{M}$ & 1.31 \\
(Zilly et al., 2017) - RHN (depth 10) & $21 \mathrm{M}$ & 1.30 \\
(Zilly et al., 2017) - Large RHN & $46 \mathrm{M}$ & 1.270 \\
(Mujika et al., 2017) - FS-LSTM-4 & $27 \mathrm{M}$ & 1.277 \\
(Mujika et al., 2017) - Large FS-LSTM-4 & $47 \mathrm{M}$ & 1.245 \\
(Merity et al., 2018a) - AWD-LSTM & $47 \mathrm{M}$ & 1.232 \\
\hline Our Method & & \\
\hline AWD-LSTM (Ours) & $47 \mathrm{M}$ & 1.257 \\
AWD-LSTM+PDR & $47 \mathrm{M}$ & 1.245 \\
\hline
\end{tabular}

Table 5: Bits-per-character on Enwik8 test set.

\begin{tabular}{lll}
\hline & PTB Valid & WT2 Valid \\
\hline AWD-LSTM (NoReg) & 108.6 & 142.7 \\
AWD-LSTM (NoReg) + PDR & 106.2 & 137.6 \\
\hline
\end{tabular}

Table 6: Validation perplexities for AWD-LSTM without any regularization and with only PDR.

our best models on the PTB and WT2 validation sets. The NLL values are significantly peaked near 0 , which means that the past decoding operation is able to decode significant amount of information about the last token in the context.

To investigate the effect of hyperparameters on PDR, we pick 60 sets of random hyperparameters 


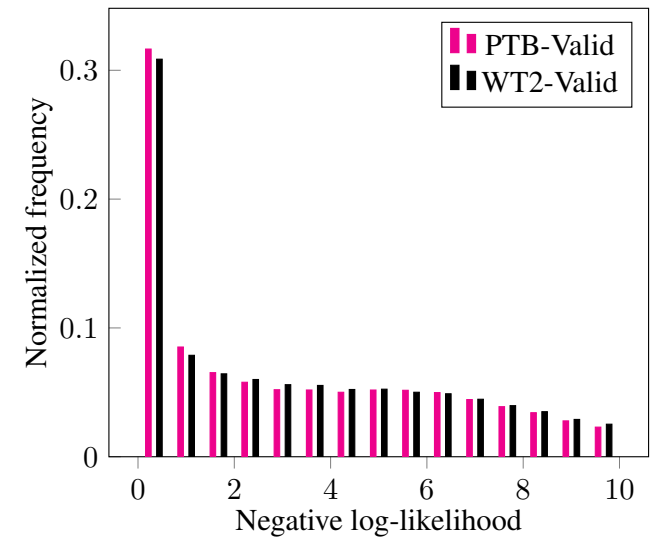

(a) Histogram of the NLL of $x_{t}$ in the past decoded (b) Histogram of validation perplexities on PTB for a vector $\mathbf{w}_{t}^{r}$.

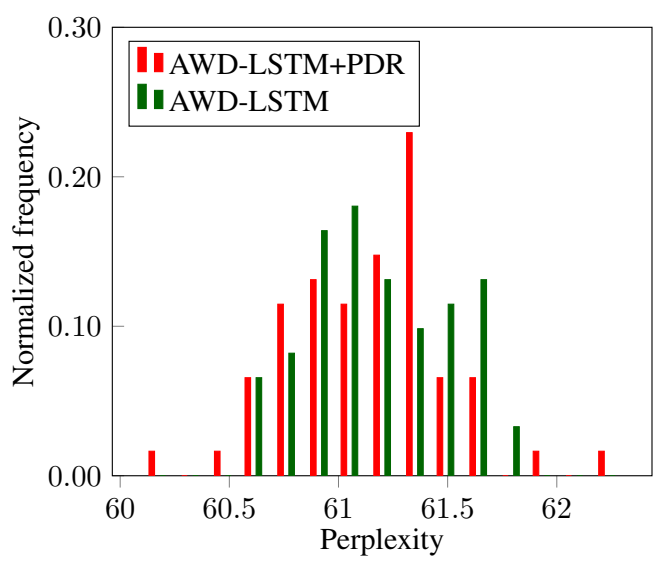

set of different hyperparameters.

Figure 1: Context token NLL for AWD-LSTM+PDR and comparison with AWD-LSTM.

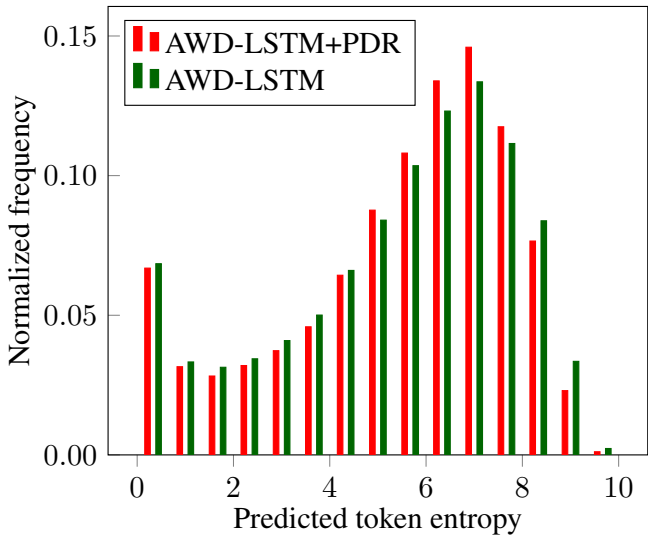

(a) Histogram of entropies of $\mathbf{w}_{t+1}$ for PTB valid.

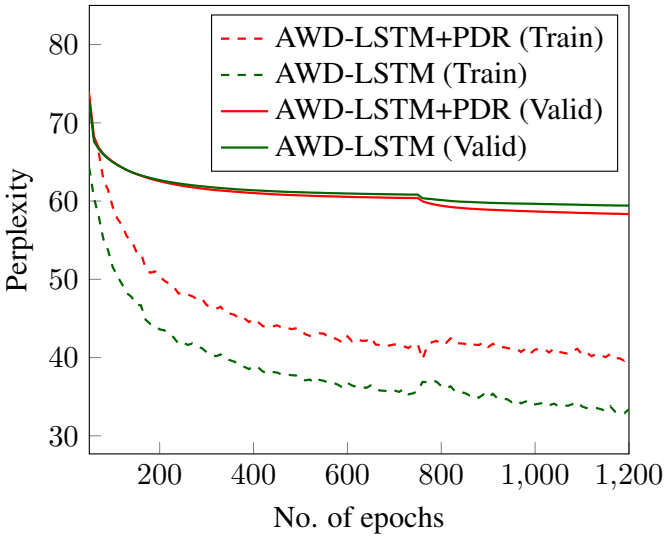

(b) Training curves on PTB showing perplexity. The kink in the middle represents the start of finetuning.

Figure 2: Comparison between AWD-LSTM+PDR and AWD-LSTM.

in the vicinity of those reported by (Merity et al., 2018b) and compute the validation set perplexity after training (without finetuning) on PTB, for both AWD-LSTM+PDR and AWD-LSTM. Their histograms are plotted in Fig.1(b). The perplexities for models with PDR are distributed slightly to the left of those without PDR. There appears to be more instances of perplexities in the higher range for models without PDR. Note that there are certainly hyperparameter settings where adding PDR leads to lower validation complexity, as is generally the case for any regularization method.

\subsection{Comparison with AWD-LSTM}

To show the qualitative difference between AWDLSTM+PDR and AWD-LSTM, in Fig.2(a), we plot a histogram of the entropy of the predicted next token distribution $\mathbf{w}_{t+1}$ for all the tokens in the validation set of PTB achieved by their respective best models. The distributions for the two models is slightly different, with some identifiable patterns. The use of PDR has the effect of reducing the entropy of the predicted distribution when it is in the higher range of 8 and above, pushing it into the range of 5-8. This shows that one way PDR biases the language model is by reducing the entropy of the predicted next token distribution. Indeed, one way to reduce the cross-entropy between $x_{t}$ and $\mathbf{w}_{t}^{r}$ is by making $\mathbf{w}_{t+1}$ less spread out in Eq.(5). This tends to benefit the language model when the predictions are correct.

We also compare the training curves for the two models in Fig.2(b) on PTB. Although the two models use slightly different hyperparame- 


\begin{tabular}{l|cc|cc}
\hline \multirow{2}{*}{ Model } & \multicolumn{2}{|c|}{ PTB } & \multicolumn{2}{c}{ WT2 } \\
& Valid & Test & Valid & Test \\
\hline AWD-LSTM+PDR & 57.9 & 55.6 & 66.5 & 63.5 \\
- finetune & 60.4 & 58.0 & 68.5 & 65.6 \\
\hline - LSTM output dropout & 67.6 & 65.4 & 75.4 & 72.1 \\
- LSTM layer dropout & 68.1 & 65.8 & 73.7 & 70.4 \\
- embedding dropout & 63.9 & 61.4 & 77.1 & 73.6 \\
- word dropout & 62.9 & 60.5 & 70.4 & 67.4 \\
- LSTM weight dropout & 68.4 & 65.8 & 79.0 & 75.5 \\
- alpha/beta regularization & 63.0 & 60.4 & 74.0 & 70.7 \\
- weight decay & 64.7 & 61.4 & 72.5 & 68.9 \\
- PDR & 60.5 & 57.7 & 69.5 & 66.4 \\
\hline
\end{tabular}

Table 7: Ablation experiments on the PTB and WT2 validation and test sets.

ters, the regularization effect of PDR is apparent with a lower validation perplexity but higher training perplexity. The corresponding trends shown in Fig.2(a,b) for WT2 have similar characteristics.

\subsection{Ablation Studies}

We perform a set of ablation experiments on the best AWD-LSTM+PDR models for PTB and WT2 to understand the relative contribution of PDR and the other regularizations used in the model. The results are shown in Table 7. In both cases, PDR has a significant effect in decreasing the validation set performance, albeit lesser than the other forms of regularization. This is not surprising as PDR does not act on the LSTM weights directly.

\section{Related Work}

Our proposed Past Decode Regularization method builds on the work of using sophisticated regularization techniques to train LSTMs for language modeling. In particular, the AWD-LSTM model achieves state-of-the-art performance with a single softmax on the four datasets considered in this paper (Merity et al., 2018b,a). (Melis et al., 2018) also achieve similar results with highly regularized LSTMs. By addressing the so-called softmax bottleneck in single softmax models, (Yang et al., 2017) use a mixture-of-softmaxes to achieve significantly lower perplexities. PDR utilizes the symmetry between the inputs and outputs of a language model, a fact that is also exploited in weight tying (Inan et al., 2016; Press and Wolf, 2017). Our method can be used with untied weights as well. Although motivated by language modeling, PDR can also be applied to seq2seq models with shared input-output vocabularies, such as those used for text summarization and neural machine translation (with byte pair encoding of words) (Press and Wolf, 2017). Regularizing the training of an LSTM by combining the main objective function with auxiliary tasks has been successfully applied to several tasks in NLP (Radford et al., 2018; Rei, 2017). In fact, a popular choice for the auxiliary task is language modeling itself. This in turn is related to multi-task learning (Collobert and Weston, 2008).

Specialized architectures like Recurrent Highway Networks (Zilly et al., 2017) and NAS (Zoph and Le, 2016) have been successfully used to achieve competitive performance in language modeling. The former makes the hidden-tohidden transition function more complex allowing for more refined information flow. Such architectures are especially important for character level language modeling where strong results have been shown using Fast-Slow RNNs (Mujika et al., 2017), a two level architecture where the slowly changing recurrent network tries to capture more long range dependencies. The use of historical information can greatly help language models deal with long range dependencies as shown by (Merity et al., 2016; Krause et al., 2018; Rae et al., 2018). In a recent paper, (Gong et al., 2018) achieve improved performance for language modeling by using frequency agnostic word embeddings (called FRAGE), a technique that is orthogonal to PDR and can be combined with it.

\section{Conclusion}

We propose a new Past Decode Regularization (PDR) method for language modeling that exploits the input-output symmetry in each step to decode the last token in the context from the predicted next token distribution. We empirically show reductions in perplexity on several benchmark datasets as compared to strong highly reg- 
ularized baseline models. Future work includes exploring the application of PDR to other seq2seq models that have a similar input-output symmetry. Also, it will be worthwhile to ascertain the efficacy of PDR for language models using Transformers and in combination with FRAGE embeddings.

\section{References}

Ciprian Chelba, Tomas Mikolov, Mike Schuster, Qi Ge, Thorsten Brants, and Phillipp Koehn. 2014. One billion word benchmark for measuring progress in statistical language modeling. In INTERSPEECH.

Junyoung Chung, Sungjin Ahn, and Yoshua Bengio. 2016. Hierarchical multiscale recurrent neural networks. CoRR, abs/1609.01704.

Ronan Collobert and Jason Weston. 2008. A unified architecture for natural language processing: deep neural networks with multitask learning. In ICML.

Yarin Gal and Zoubin Ghahramani. 2016. A theoretically grounded application of dropout in recurrent neural networks. In NIPS.

Cheng Yue Gong, Di He, Xu Tan, Tao Qin, Liwei Wang, and Tie-Yan Liu. 2018. Frage: Frequency-agnostic word representation. CoRR, abs/1809.06858.

Edouard Grave, Armand Joulin, and Nicolas Usunier. 2016. Improving neural language models with a continuous cache. CoRR, abs/1612.04426.

David Ha, Andrew M. Dai, and Quoc V. Le. 2016. Hypernetworks. CoRR, abs/1609.09106.

Sepp Hochreiter and Jürgen Schmidhuber. 1997. Long short-term memory. Neural computation, 9(8):1735-1780.

M. Hutter. 2018. The human knowledge compression contest.

Hakan Inan, Khashayar Khosravi, and Richard Socher. 2016. Tying word vectors and word classifiers: A loss framework for language modeling. CoRR, abs/1611.01462.

Ben Krause, Emmanuel Kahembwe, Iain Murray, and Steve Renals. 2018. Dynamic evaluation of neural sequence models. In ICML.

David Krueger, Tegan Maharaj, János Kramár, Mohammad Pezeshki, Nicolas Ballas, Nan Rosemary Ke, Anirudh Goyal, Yoshua Bengio, Hugo Larochelle, Aaron C. Courville, and Christopher Joseph Pal. 2016. Zoneout: Regularizing rnns by randomly preserving hidden activations. CoRR, abs/1606.01305.

Gábor Melis, Chris Dyer, and Phil Blunsom. 2018. On the state of the art of evaluation in neural language models. In ICLR.
Stephen Merity, Nitish Shirish Keskar, and Richard Socher. 2018a. An analysis of neural language modeling at multiple scales. CoRR, abs/1803.08240.

Stephen Merity, Nitish Shirish Keskar, and Richard Socher. 2018b. Regularizing and optimizing LSTM language models. In ICLR.

Stephen Merity, Caiming Xiong, James Bradbury, and Richard Socher. 2016. Pointer sentinel mixture models. CoRR, abs/1609.07843.

Tomas Mikolov, Martin Karafiát, Lukás Burget, Jan Cernocký, and Sanjeev Khudanpur. 2010. Recurrent neural network based language model. In $I N$ TERSPEECH.

Asier Mujika, Florian Meier, and Angelika Steger. 2017. Fast-slow recurrent neural networks. In NIPS.

Ofir Press and Lior Wolf. 2017. Using the output embedding to improve language models. In $E A C L$.

Alec Radford, Karthik Narasimhan, Tim Salimans, and Ilya Sutskever. 2018. Improving language understanding by generative pre-training.

Jack W. Rae, Chris Dyer, Peter Dayan, and Timothy P. Lillicrap. 2018. Fast parametric learning with activation memorization. In ICML.

Marek Rei. 2017. Semi-supervised multitask learning for sequence labeling. In $A C L$.

Kamil Rocki, Tomasz Kornuta, and Tegan Maharaj. 2016. Surprisal-driven zoneout. CoRR, abs/1610.07675.

Nitish Srivastava, Geoffrey E. Hinton, Alex Krizhevsky, Ilya Sutskever, and Ruslan Salakhutdinov. 2014. Dropout: a simple way to prevent neural networks from overfitting. Journal of Machine Learning Research, 15:1929-1958.

Li Wan, Matthew D. Zeiler, Sixin Zhang, Yann LeCun, and Rob Fergus. 2013. Regularization of neural networks using dropconnect. In ICML.

Zhilin Yang, Zihang Dai, Ruslan Salakhutdinov, and William W. Cohen. 2017. Breaking the softmax bottleneck: A high-rank rnn language model. CoRR, abs/1711.03953.

Wojciech Zaremba, Ilya Sutskever, and Oriol Vinyals. 2014. Recurrent neural network regularization. CoRR, abs/1409.2329.

Julian G. Zilly, Rupesh Kumar Srivastava, Jan Koutník, and Jürgen Schmidhuber. 2017. Recurrent highway networks. In ICML.

Barret Zoph and Quoc V. Le. 2016. Neural architecture search with reinforcement learning. CoRR, abs/1611.01578. 